\title{
Study of Correlative Evaporation and Ion Dissociation in Atom Probe Data
}

\author{
Yimeng Chen, Brian Geiser, Ed Oltman, Katherine Rice, Robert Ulfig and Ty Prosa
}

CAMECA Instruments, Inc, Madison, Wisconsin, United States

In atom probe tomography, a multi-hit event is defined as more than one ion detected during a single laser or voltage pulse. The multiplicity is the number of recorded ions for a single pulse. For some materials and analysis conditions, multi-hit events may be present in as many as $60 \%$ of recorded pulses. Common examples of applications with high multiplicity are oxides, III/V compounds, carbon in steels and both boron and carbon in silicon [1]. In general, the compounds made of ionic or covalent bonding are known to produce multi-hit events at much higher rates than predicted by random (uncorrelated) evaporations. Ion dissociation and post-ionization are the main factors that are thought to contribute to high multiplicity events. Non-random (correlated) evaporations can result in non-detectable ions (e.g. may not even appear as multi-hit events) that can bias compositional measurement. In some cases, accurate compositional measurements cannot be made without correction factors.

Correlated ion analysis, colloquially known as a "Saxey plots," is a powerful method to study the correlated evaporation events in atom probe data [2]. Tracks of dissociated species exhibit a "negative slope" in the Saxey plot. The corresponding dissociation mechanism can be then interpreted from the plot. Gault et al. also reported evidence of neutral species and measured energy deficits associated with dissociation using correlated ion analysis [3]. Correlated analysis also enables exploring the neutral species from the dissociation process where datasets from both the straight flight path and the reflectron system will be compared to better understand the behavior of neutral species.

In this work, a range of materials were chosen to study the correlated evaporations and molecular dissociation by systematically varying the analysis conditions. Specimens are prepared using focused ion beam milling under the same milling process to minimize any geometric differences in the tip shape. Automated data acquisition with preset analysis conditions are used to increase productivity and consistency, enabling large-scale studies. Codes to perform the correlated ion analysis will be incorporated into AP Suite $6^{\mathrm{TM}}$ from CAMECA ${ }^{\circledR}$. Ultimately, methods and factors that are necessary in correcting composition measurement will be discussed.

\section{References}

[1] Rolander, U. \& Andren, H.-O. On Atom-Probe Analysis of Cubic MX-Type Carbides and Carbonitrides. Journal de Physique 49, 299-304 (1988).

[2] Saxey, D. W. "Correlated Ion Analysis and the Interpretation of Atom Probe Mass Spectra." Ultramicroscopy 111 (2011): 473-79.

[3] Gault, Baptiste, David W. Saxey, Michael W. Ashton, Susan B. Sinnott, Ann N. Chiaramonti, Michael P. Moody, and Daniel K. Schreiber. "Behavior of Molecules and Molecular Ions near a Field Emitter." New Journal of Physics 18, no. 3 (2016): 033031. 